\title{
ФИЗИЧЕСКАЯ ХИМИЯ
}

УДК 544.032 .5

DOI 10.26456/vtchem2021.3.1

\section{МОЛЕКУЛЯРНЫЙ ПЕРЕКЛЮЧАТЕЛЬ НА ОСНОВЕ БИТИОФЕН-АЗОБЕНЗОЛА: КАК УПРАВЛЯТЬ ПРОВОДИМОСТЬЮ СЛОЯ С ПОМОЩЬЮ СВЕТА}

\begin{abstract}
В.А. Савченко, ${ }^{1}$ О.А. Гуськова ${ }^{1,2}$
${ }^{1}$ Института полимерных исследований ассоииации Лейбница, институт теории полимеров, Дрезден, Германия

${ }^{2}$ Дрезденский технический университет,

Дрезденский центр вычислительного материаловедения, Дрезден, Германия

Молекулярные переключатели на основе азобензола (азо) являются светочувствительными молекулами, которые могут переключаться между двумя конфигурационными состояниями под действием света. Светочувствительные азо-монослои можно использовать для модуляции работы выхода, то есть они влияют на свойства электродов. В данной работе мы отвечаем на вопрос, что происходит со структурами, электронными свойствами и перераспределением заряда в монослоях азобитиофена $(a з о-б m)$ в зависимости от светового стимула, используя теорию функционала плотности. Моделируются два типа переключателей, различающихся расположением $а з о$ и бт от группы пришивки молекулы к

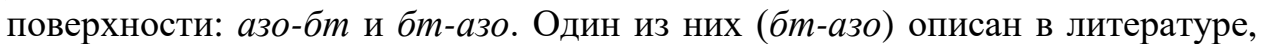
другой же является продуктом молекулярного дизайна. Мы описываем транс- и цис-изомеры для каждого переключателя, находящегося в контакте с кластером золота. Наше моделирование объясняет гигантское соотношение в проводимости ON/OFF-состояний при воздействии УФизлучения на монослой улучшенной электронной связью между цисизомерами (состояние ON) и кластером золота. Транс-изомеры же (OFF состояние) моделируемых переключателей играют роль изоляторов. Кроме того, мы показываем, какие именно свойства улучшаются после молекулярного дизайна. Данное исследование открывает новые возможности в разработке инновационных модификаций поверхности электродов.

Ключевые слова: азобензол, изомеризачия, фоточувствительность, проводимость, метод функционала плотности, привитой монослой, кластер золота $А u_{10}$
\end{abstract}

\section{Введение и актуальность проблемы}

Постоянно развивающееся современное вычислительное оборудование от процессоров до запоминающих устройств основано на электронных компонентах чрезвычайно малых размеров и высокой энергоэффективности. Молекулярные туннельные переходы в 
подобных устройствах, в которых используются свойства переноса заряда, имеют в этом контексте значительный потенциал, потому что изменения на атомарном масштабе могут привести к существенным эффектам на макроуровне всего устройства.

Материалы для молекулярных туннелей являются не просто многокомпонентными структурами. Они подбираются таким образом, чтобы регулировать и подстраивать свои свойства в зависимости от внешнего воздействия. На сегодняшний день наибольшие применимость и влияние в качестве внешнего триггера имеет свет [1].

Для того, чтобы обеспечить светочувствительность активного компонента электронного устройства (например, тонких пленок) небольшие молекулы, называемые фотохромами и претерпевающие обратимую изомеризацию под действием света между по крайней мере двумя состояниями, должны быть структурно встроены в органические полупроводниковые материалы - сопряженные полимеры, олигомеры. Первая проблема, с которой сталкиваются ученые, - выбор подходящей фотохромной молекулы. Ведь, действительно, фотохромы включают большую группу соединений [2]. Однако для применения в молекулярной электронике подходят только те молекулы, фотоизомеры которых термически стабильны, а их свойства (такие, как геометрия, орбитальные уровни, ширина запрещенной зоны, окислительновосстановительные потенциалы и оптические характеристики) претерпевают при изомеризации серьезные изменения. Среди прочих, $[3,4]$ наиболее подходящими фотохромами являются азобензолы (азо), поскольку они удовлетворяют большинству перечисленных условий.

В литературе было показано, что резкая перестройка молекулярной структуры из плоского транс- в изогнутое цис-азо состояние при УФ-облучении в первую очередь приводит к изменению физико-механических свойств полупроводниковых материалов. Поэтому фотопереключаемые материалы на основе азо представляют интерес для приведения в действие органических электронных устройств [5]. Ожидается, что инициируемая светом реорганизация, сопровождаемая, кроме того, большим изменением дипольного момента изомеров, изменит энергетический ландшафт зарядов, переносимых внутри полупроводникового слоя [6]. По этой причине азопереключаемые материалы могут обеспечивать фотоуправление межмолекулярным электронным взаимодействием и путями передачи энергии, то есть, тем, как в тонких полупроводниковых пленках носители заряда передвигаются в пространстве от одной молекулы к другой $[7,8]$. 


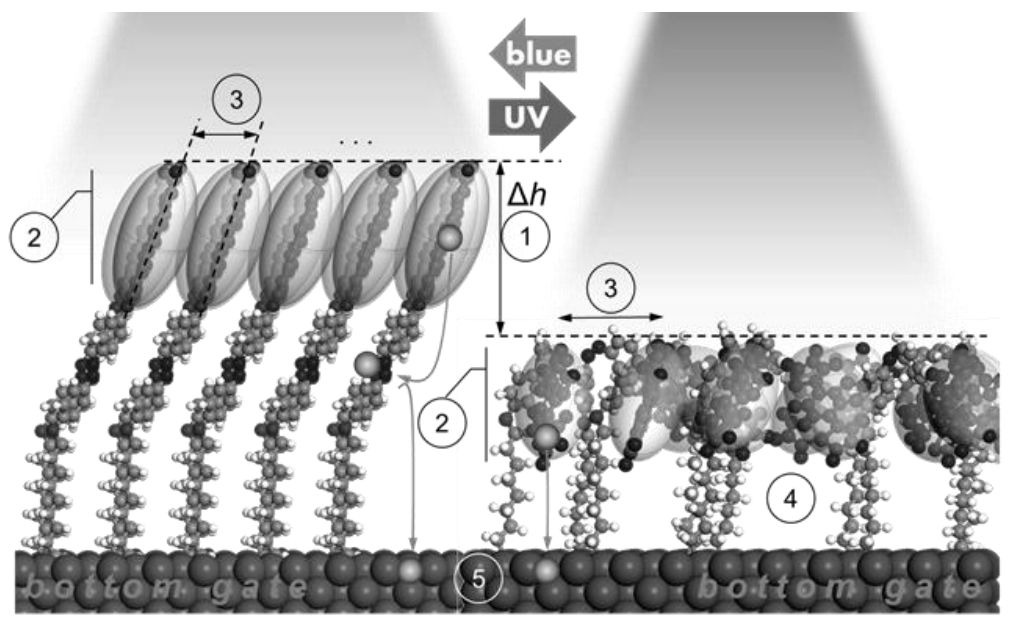

Р и с . 1. Молекулярный туннельный переход (верхний электрод не показан) и его полная оптическая перестройка при облучении видимым $\lambda=480$ нм (слева) и УФсветом $\lambda=360$ нм (справа). Здесь схематически показан монослой из привитых азомолекул. Их структурная формула приведена на Рис. 2. Изменяющимися свойствами являются толщина слоя ( $\Delta h$ - разница высот транс- и цис-слоев) (1), упаковка (2), тип и сила межмолекурярного взаимодействия (3), конформация углеводородной цепи (4), с помощью которой молекулы химически пришиты к нижнему электроду (5). Эллипсоидом здесь показан олигомер сопряженного полимера

Схематическое представление подобной системы дано на Рис. 1. Здесь пространство между двумя электродами заполнено монослоем из молекул, химические формулы которых показаны на Рис. 2. Эти молекулы, описанная в литературе бт-азо [9-15], а также ее структурный изомер азо-бт (пример молекулярного дизайна). Они и являются объектами нашего исследования. Синтез бт-азо подробно описан Karpe и др. [9]. В обеих молекулах азобензольный блок сочетается с битиофеном. Такое сочетание может повлиять на степень сопряжения, дипольный момент и жесткость остова, а также привнести новые функции, такие как более выраженный стекинг между сопряженными участками молекул, изменение реакции системы на световой стимул (то есть ускорение коллективного транс-цис переключения при облучении) или, наконец, значительное уширение спектров оптического поглощения.

В экспериментах бт-азо молекулы пришивались с помощью тиольной группы на поверхность плоского золотого электрода (111 Au) (позже на поверхность кобальта [11]) или золотых наночастиц $[9,10,12$ 15]. Вторым, верхним электродом, выступал проводящий наконечник зонда, а методом исследования была электропроводящая атомносиловая микроскопия. Было установлено, что при облучении ультрафиолетом проводимость между электродами увеличивалась в 700 раз [9] (в других измерениях это отношение было еще выше [10]), а при 
обратном переводе молекул в транс-состояние облучением синим светом проводимость слоя была близка к нулю. Таким образом, цис- и транс-изомеры были охарактеризованы как ON/OFF (ВКЛ/ВЫКЛ) состояния.

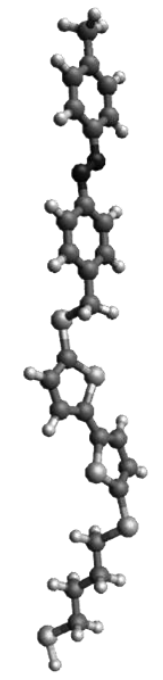

(a) бт-азо

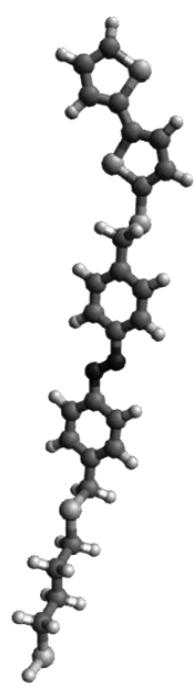

(б) $а з о-б m$

Р и с . 2. Молекулярный переключатель (а) $E$ 1-(4-((5'-(4-меркаптобутилсульфанил)-2,2'битиофен-5-сульфанил)метил)фенил)-2-pтолилдиазен, сокращенно бт-азо (аббревиатура указывает на порядок блоков, считая от тиольной группы-пришивки: битиофен-азобензол) описан в литературе [915], в то время как его структурный изомер (б) азо-бm (азобензол-битиофен) описывается здесь впервые и представляет собой результат молекулярного дизайна. Обе молекулы находятся в транс-конфигурации азо-группы.

Авторами были приведены несколько объяснений подобного переключения $[9,10]$, главное из которых сводится к тому, что изменения проводимости связаны, в основном, с длиной туннельного барьера между верхним зондом/электродом и нижним электродом, то есть, с конфигурационными изменениями бт-азо. Другие механизмы уже рассматривались при помощи компьютерного моделирования. Так, например, Lenfant и др. [13] смоделировали изолированные транс- и цис-изомеры этого переключателя, применяя теорию функционала плотности (ТФП) с параметрами B3LYP/6-311G, и рассчитали спектры оптического поглощения для оптимизированных изомеров в вакууме, используя динамическую модификацию ТФП. Те же самые бт-азо изомеры в контакте с верхним электродом, расположенным на 0.2 нм выше молекулы и смоделированным как кластер $\mathrm{Au}_{11}$, были описаны с использованием ТФП с параметрами B3LYP и базисными наборами 6$31 \mathrm{G}$ и LANL2DZ для органических и неорганических частей системы соответственно [10]. Главный теоретический результат заключался в том, что высокое отношение цис/транс-проводимости скорее связано с изменениями как во взаимодействии молекула-электрод, так и в электронной структуре соединения металл-молекулы-металл, чем с разницей в толщине монослоя при разном облучении.

Целью данной работы является объяснение наблюдаемых в эксперименте процессов для бт-азо изомеров, а также предсказание того, какими свойствами в аналогичных условиях будет обладать 
структурный изомер азо-бт. Для этого мы опишем струкрурные и электронные свойства изолированных изомеров, а также их комплексов с кластером золота, имитирующим кончик зонда атомно-силового микроскопа. Мы предполагаем, что верхний механический электрод, контактируя с разными атомными группами бт-азо или азо-бm, будет по-разному участвовать в делокализации электронной плотности и переносе заряда от переключателя к электроду.

\section{Методика расчетов}

В литературе существует множество вычислительных подходов к описанию азобензолов или азо-содержащих соединений, привитых к различным поверхностям. В наших предыдущих исследованиях использовались теоретические и вычислительные методы в диапазоне от $a b$ initio и атомистической молекулярной динамики до крупнозернистого моделирования и термодинамической теории [16-21]. Цель настоящего исследования - предсказать, как конфигурационные перестройки и молекулярный дизайн влияют на структурные, электронные и транспортные свойства молекулярных переключателей и их комплексов с кластером золота, имитирующем кончик зонда. Поэтому методология включает использование подходов ТФП и ее динамической версии для изолированных изомеров и комплексов.

Оптимизированные геометрии изолированных бт-азо и азо-бт изомеров получены ТФП с использованием функционала B3LYP с базисным набором 6-31G(d,p) в программе GAUSSIAN09 Rev.C01 [22] Эта комбинация широко и успешно используется в исследованиях азосодержащих молекул, демонстрируя согласие расчетных и экспериментальных данных [23,24]. Частотный анализ применялся для подтверждения того, что молекулы в их оптимизированной геометрии находятся в локальном минимуме на поверхности потенциальной энергии: вывод основывается на отсутствии мнимых частот в ИКспектрах. Необходимо также отметить, что битиофен в составе молекул моделировался только в транс-конформации, а менее стабильный цисконформер [25,26] не рассматривался. Как следует из литературы, комбинация B3LYP/6-31G(d,p) дает адекватные предсказания свойств азобензолов в УФ и видимой областях [27,28]. Учет эффекта растворителя (дихлорметан, как в экспериментах Каrpe и др. [9]) проводился с помощью модели поляризуемого континуума в формализме интегрального уравнения (IEF-PCM) [29].

Кластер золота, состоящий из 10 атомов с фиксированными координатами, представляет собой тетраэдр с длиной ребра $5.68 \AA$ и расстоянием между соседними атомами $2.88 \AA$. Комплексы кластера золота и органической молекулы моделировались с применением дубльзета базисного набора с эффективным потенциалом остова LANL2DZ для атомов золота и указанным выше потенциалом, и базисным 
набором для бт-азо и азо-бт. Зонд располагался над разными частями четырех изомеров и оставался неизменяемым, в то время как геометрия переключателей подстраивалась во время оптимизации под неподвижный кластер. Начальное расстояние между зондом и переключателем задавалось равным $2 \AA$, как в первых работах Smaali и др. [10] и Lenfant и др. [13].

\section{Результаты расчетов и их обсуждение}

Наиболее важные свойства изомеров исследуемых переключателей приведены в Табл. 1. Из представленных данных следует, что бт-азо и азо-бт характеризуются разными значениями $\Delta h$ : $6.05 \AA$ и $14.69 \AA$, соответственно. Интересно отметить, что первое значение бт-азо близко к литературным данным для того же соединения [13]: 28.6 (транс) и $23.5 \AA$ (цис) с $\Delta h=5.1 \AA ̊$. Наиболее сильное реструктурирование слоя, отмеченное на Рис. 1 , и уменьшение в два раза ширины перехода между электродами предсказано для азобm переключателя. Расположение светочувствительной азо-группы ближе к плоскости электрода приводит к тому, что геометрия молекулы при облучении изменяется практически полностью, и только алкильный спейсер остается незатронутым. Изменение молекулярной геометрии отражается и на статических дипольных моментах. Из литературы известно, что даже умеренные изменения направления и величины молекулярных диполей важны для изменения магнитных свойств контактов [30]. Более того, поверхностный диполь является одним из параметров, определяющих работу выхода электрона $\phi$. В соответствии с классическим уравнением Гельмгольца [31], изменение рабочей функции определяется как

$$
\Delta \phi=\frac{\mu_{z}}{\varepsilon_{0} S}
$$

где $\mu_{z}$ - дипольный момент, перпендикулярный поверхности и $S-$ площадь поверхности, занимаемая одной молекулой.

Дипольные моменты не только практически в два раза увеличивают свои значения при УФ облучении, но и меняют свое направление: для транс-изомеров векторы направлены от тиольной группы, в то время как для цис-состояний векторы моментов указывают к поверхности пришивки монослоя. Необходимо подчеркнуть, что в предыдущих работах по моделированию бт-азо авторы приводят, вопервых, различные, а во-вторых, завышенные значения дипольных моментов: например, Lenfant и др. [10] получили $\mu_{\text {цис }}=4.9$ и $\mu_{\text {транс }}=1.8 \mathrm{D}$ (B3LYP/6-31G), и тот же авторский коллектив [13] несколькими годами позже опубликовал значения $\mu_{\text {цис }}=8.01$ и $\mu_{\text {транс }}=5.15 \mathrm{D}$ (B3LYP/6-311G). При этом значения высот монослоев, состоящих из транс- или цисизомеров бт-азо совпадают с нашими результатами, как упоминалось выше. 
Т аблица 1

Свойства изомеров бт-азо и азо-бт, полученные из анализа оптимизированных геометрий молекул в дихлорэтане

\begin{tabular}{|c|c|c|c|c|c|c|c|}
\hline Свойства & Изомер & $\begin{array}{l}\text { Высота } \\
\text { слоя } \\
{[\AA]}\end{array}$ & $\mid \begin{array}{ll}\text { Дипольны } \\
\text { й } \\
\mu,[D]\end{array}$ & 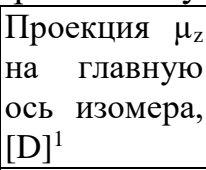 & $\begin{array}{l}\mathrm{z} \\
\mathrm{o} \\
\text { Tорсионные } \\
\text { углы } \\
\gamma_{\text {бт }},\left[^{\circ}\right]^{2}\end{array}$ & $\begin{array}{l}\text { Объем } \\
\text { молекулы } \\
V,\left[\AA^{3}\right]^{3}\end{array}$ & $\begin{array}{l}E_{\mathrm{g}} \\
{[\mathrm{eV}]}\end{array}$ \\
\hline \multirow{2}{*}{ бт-азо } & транс & 29.54 & 1.34 & 1.22 & $\begin{array}{l}179.9 \\
163.8 \\
\end{array}$ & 2655 & 2.28 \\
\hline & цис & 23.49 & -3.22 & -2.51 & $\begin{array}{l}9.9 \\
160.9\end{array}$ & 2615 & 2.30 \\
\hline \multirow{2}{*}{ азо-бт } & транс & 29.89 & 1.67 & 0.76 & $\begin{array}{l}179.9 \\
158.0\end{array}$ & 2653 & 2.25 \\
\hline & цис & 15.20 & -3.52 & -0.14 & $\begin{array}{l}9.6 \\
158.4 \\
\end{array}$ & 2610 & 2.29 \\
\hline
\end{tabular}

${ }^{1}$ Статические диполи молекул проецируются здесь на главную ось изомера (обычно на нормаль к поверхности нижнего электрода [30]). Знак дипольного момента положительный, если его направление совпадает с направлением Z оси молекулы, считая от тиольной группы-пришивки, и отрицательным vice versa.

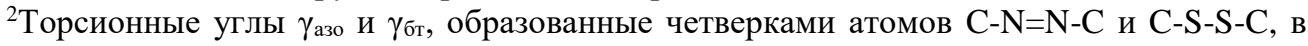
азобензоле и битиофене, соответственно.

${ }^{3}$ Объем молекул рассчитан как объем внутри поверхности, доступной растворителю. Радиус молекулы дихлорметана для расчета принимался равным $2.52 \AA$.

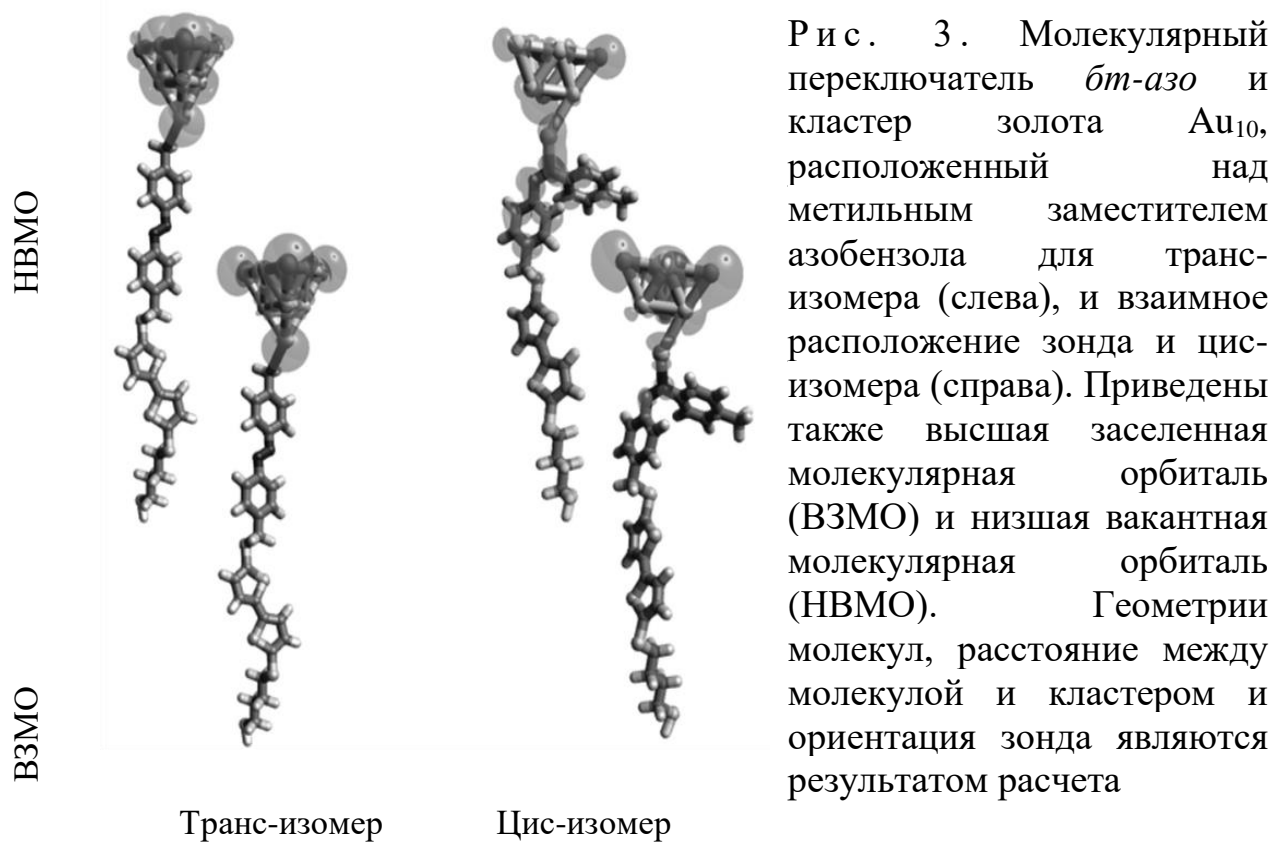

Торсионные углы, характеризующие взаимную ориентацию бензольных колец в азо, незначительно отличаются от величин для транс- и цис-изомеров азобензола и различных замещенных азобензолов [17-20]. Нами подтверждается копланарность колец для транс-изомера, а также значения около $10^{\circ}$ для цис-формы обоих переключателей. Транс-битиофеновый фрагмент, однако, показывает такие значения 
торсионных углов, которые в среднем более чем на $20^{\circ}$ отклоняются от планарности по сравнению с незамещенным битиофеном [25]. Наиболее вероятная причина состоит во влиянии атомов серы (мезомерный эффект от неподеленных электронных пар атомов серы), которые замещают водороды в одном или двух тиофеновых кольцах. Отклонение от планарности простейшего серазамещенного 2,2'битиофен-5-тиола было установлено в компьютерном моделировании Sato и др. [32].

Молекулярный объем, как следует из Табл. 1, незначительно уменьшается при изомеризации для двух переключателей, а также не зависит от порядка блоков бт-азо или азо-бт. Однако следует помнить, что для привитого монослоя разница в 40-43 $\AA^{3}$ в объеме молекулы, равно как и латеральные стерические конфликты соседних молекул могут иметь существенное влияние на изомеризационную кинетику.

Наконец, ширина запрещенной зоны $E_{\mathrm{g}}$ практически не зависит от молекулярного дизайна и составляет $2.28 \mathrm{eV}$ для транс- и $2.30 \mathrm{eV}$ для цис- изомеров бт-азо и $2.25 \mathrm{eV}$ для транс- и $2.29 \mathrm{eV}$ для цис- изомеров азо-бт. Аналогичная тенденция, а именно незначительное уширение запрещенной зоны, было найдено в литературе [13].

Теперь рассмотрим свойства комплексов переключателей с нанокластером золота. Здесь необходимо отметить, какие системы моделировались, то есть как располагался зонд над изомерами переключателей. Все пары для бт-азо приведены на Рис. 3, а для азо-бт - на Рис. 4.

Из-за того, что азо-бт претерпевает при изомеризации значительные перестройки и потенциально может взаимодействовать с кластером разными блоками, нами рассматривается четыре варианта возможной взаимной ориентации (Рис. 4). На практике, вероятнее всего, ответ от вариантов (а)-(г) при УФ-облучении будет усреднен, но, поскольку молекулярное моделирование предоставляет возможность оценить вклад каждого такого взаимодействия по отдельности, это, несомненно, интересно рассмотреть более детально.

Начнем с анализа граничных молекулярных орбиталей (МО) бmaзо, приведенных на Рис. 3. Транс-изомер взаимодействует с зондом своим метильным заместителем, который, по сути, является изолирующей группой. Именно поэтому НВМО и ВЗМО локализованы только на кластере золота. Следовательно, никакого значительного влияния на свойства системы такой контакт не окажет. Напротив, для цис-изомера делокализация НВМО и ВЗМО включает в себя не только кластер, но и полностью азо-блок в случае НВМО, и азо-группу для ВЗМО. Это означает электронную коммуникацию между зондом и переключателем, что может отразиться, например, на перераспределении зарядов, то есть, частичном транспорте заряда, поляризации системы. 


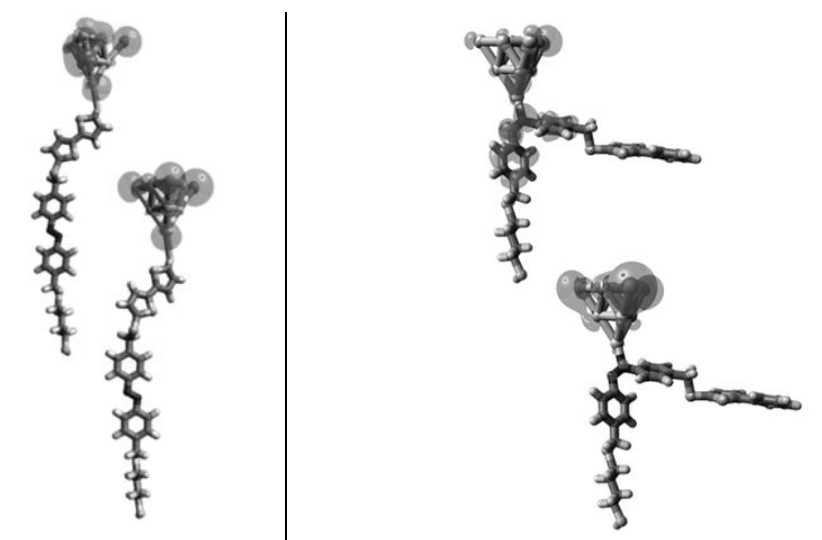

Кластер над азо-группой (a)

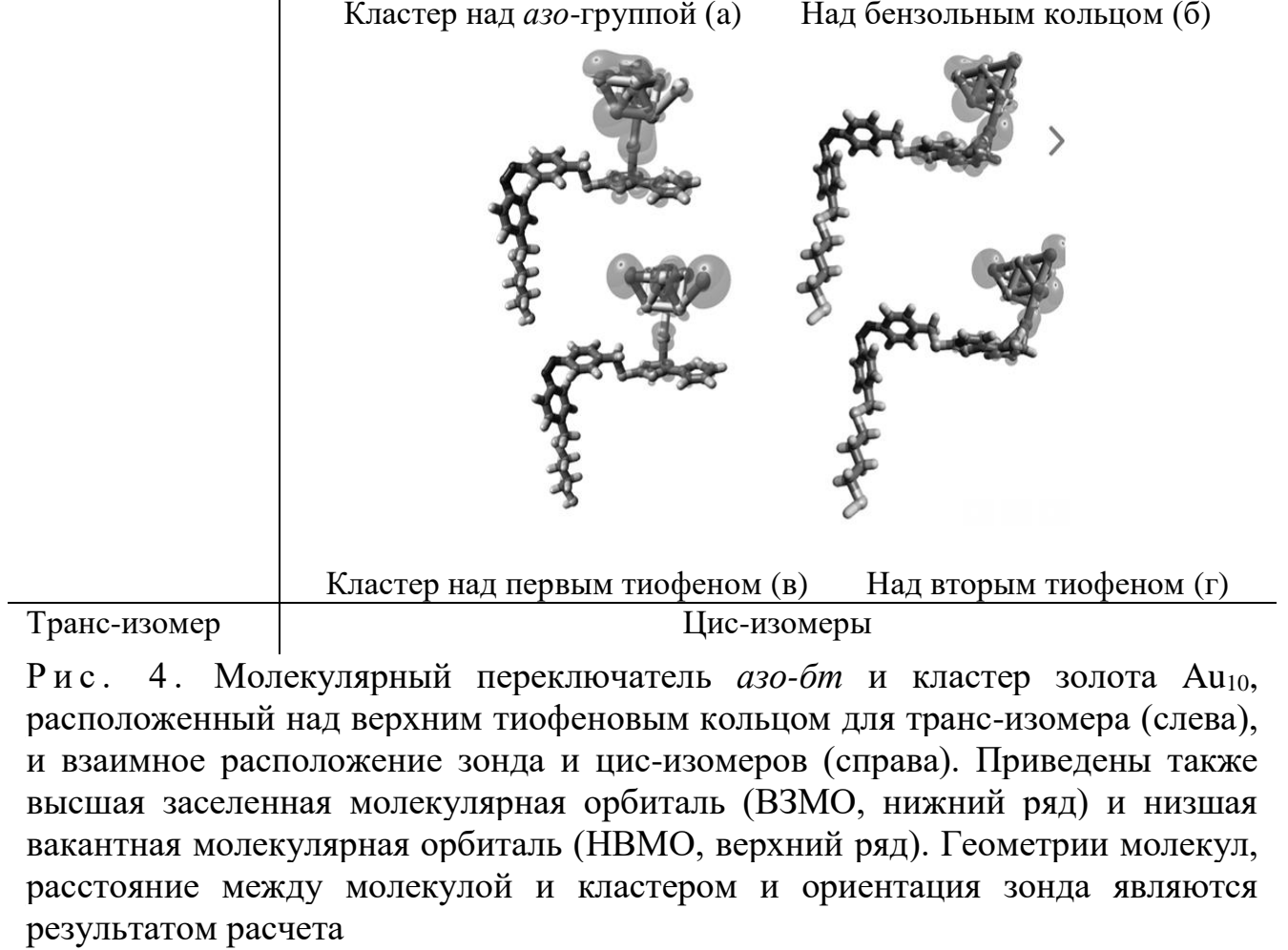

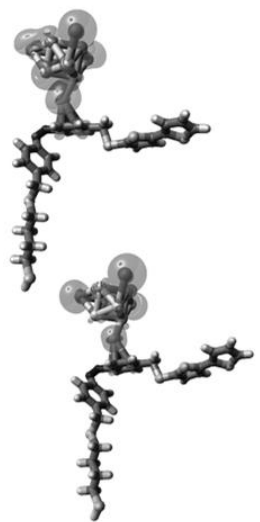

Над бензольным кольцом (б)

Для количественной оценки перераспределения заряда в комплексах применялся популяционный анализ Малликена (Табл. 2). Из данных видно, что при УФ облучении каждый цис-изомер передает $0.201 e$ зонду, а в случае транс-состояний электронного взаимодействия практически нет. Этот результат в совокупности с визуализацией граничных МО уже достаточен для объяснения наблюдаемого в эксперименте $[9,10]$ переключения проводимости слоя от состояния ON (ВКЛ) при УФ облучении до OFF(/ВЫКЛ) в темноте или при облучении синим светом. Здесь наши результаты полностью совпадают с литературными данными [10]. 
Таблица 2

Избыточный заряд на кластере золота в комплексах с изомерами бт-азо и азо-бт и магнитуда их дипольного момента

\begin{tabular}{|l|l|c|c|}
\hline $\begin{array}{l}\text { Переключатель } \\
\text { /Свойства }\end{array}$ & $\begin{array}{l}\text { Изомер } \\
\text { (Рис. 3, 4) }\end{array}$ & $\begin{array}{l}\text { Избыточный заряд на } \\
\text { кластере золота } \Delta q,[e]\end{array}$ & $\begin{array}{l}\text { Дипольный } \\
\text { комплекса } \mu_{\text {комплекс, [D] }}\end{array}$ \\
\hline \multirow{3}{*}{ бт-азо } & транс & -0.042 & 1.76 \\
\cline { 2 - 4 } & цис & -0.201 & 14.19 \\
\hline \multirow{5}{*}{ азо-бm } & транс & -0.041 & 2.01 \\
\cline { 2 - 4 } & цис (a) & -0.312 & 9.29 \\
\cline { 2 - 4 } & цис (б) & -0.251 & 13.42 \\
\cline { 2 - 4 } & цис (в) & -0.322 & 9.02 \\
\cline { 2 - 4 } & цис (г) & -0.296 & 10.54 \\
\hline
\end{tabular}

Теперь интересно сравнить, как отражается на перераспределении заряда дизайн молекулы, то есть, перестановка азобензольного и битиофенового блоков. Для транс-азо-бт вероятность передать парциальный заряд кластеру так же низка, как и для транс-бтазо, даже несмотря на то, что контакт осуществляется не через метильный заместитель, а напрямую с тиофеновым кольцом. Вероятно от того, что битиофеновый фрагмент направлен не плоскостями колец, а ребром одного из них к зонду, электронного обобществления не происходит. Все цис-состояния передают в среднем треть своего заряда кластеру золота, что больше заряда, передаваемого одной молекулой цис-бт-азо. Граничные МО для цис-азо-бт делокализуются на зонде и части молекулы. Здесь проявляется и сопряжение в битиофеновом блоке: независимо от положения (в) или (г), МО распределена по всему блоку. Кроме большей величины $\Delta h$ для азо-бm, здесь видно еще одно преимущество такого чередования блоков, а именно, большее взаимодействие между переключателем и зондом. Следует ожидать, что азо-бт переключатель может быть более эффективным, давая большие ON/OFF-соотношения проводимостей.

Величины дипольных моментов согласуются с только что описанными данными по переносу зарядов. Для транс-состояний электрический дипольный момент незначительно больше значений, указанных в Табл. 1, в то время как для цис-комплексов значения

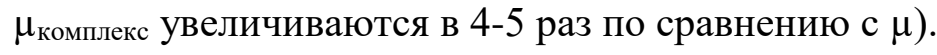




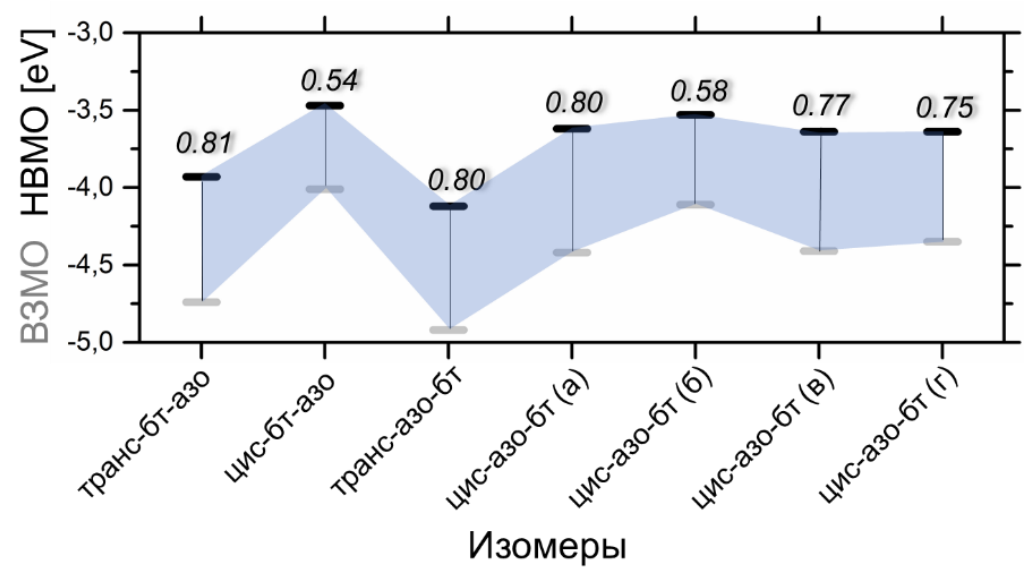

Р и с. 5. Энергии НВMO и ВЗМО и ширина запрещенной зоны $E_{\mathrm{g} \text { комплекс }}[\mathrm{eV}]$ для рассматриваемых комплексов. Значения $E_{\mathrm{g}}$ комплекс написаны курсивом

Последнее свойство, которого мы коснемся в обсуждении, это энергии граничных молекулярных орбиталей и связанные с ними

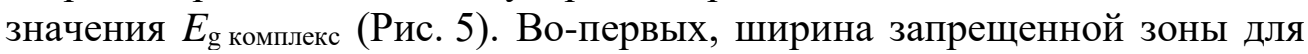
комплексов значительно меньше по сравнению $E_{\mathrm{g}}$ изолированных переключателей. Второй вывод, который наиболее важен с точки зрения применения света для управления свойствами молекулярных переходов, заключается в том, что для транс-изомеров энергии НВМО и ВЗМО близки к значению рабочей функции ф золота (около $5 \mathrm{eV}$ [33]), а при облучении ультрафиолетом работа выхода ф уменьшается в среднем на $1 \mathrm{eV}$. Сравнивая данные Рис. 5 для двух переключателей, необходимо подчеркнуть, что экспериментально описанная молекула бт-азо эффективнее снижает работу выхода.

\section{Заключение}

В данной работе с помощью расчетов методом ТФП рассчитывались свойства двух переключателей бт-азо и азо-бт, которые предполагается использовать для изменения свойств туннельных переходов с помощью облучения светом определенной длины волны. В структуру обеих молекул встроен азобензол фотохром, претерпевающий транс-цис изомеризацию при облучении ультрафиолетом. В качестве верхнего электрода моделируется кластер золота, приведенный в контакт с изомерами.

Главным выводом работы можно назвать обнаруженную причину переключения проводимости слоя при облучении, описанную в эксперименте $[9,10,13]$. Причина не только в разнице высот молекулярного слоя транс- и цис-состояний, но, что наиболее важно, электронная делокализация и перенос заряда с молекулы на зонд именно для цис-изомеров. Поэтому цис-изомеры дают многократный рост проводимости в эксперименте. Более того, нами показано, что цисизомеры бт-азо и азо-бт становятся значительно более полярными, 
особенно их комплексы, что будет снижать рабочую функцию электрода.

Несмотря на важность проведенных расчетов следует подчеркнуть, что представленные здесь системы являются до некоторой степени упрощенными. Во-первых, они не учитывают влияние нижнего электрода на систему. Между тем, после адсорбции на металле, а тем более хемисорбции, цис-транс переключение неэффективно или может и вовсе отсутствовать из-за быстрого гашения возбужденного состояния или потери транс-цис бистабильности [34]. Во-вторых, рассматривались одиночные молекулы вне монослоя, то есть без влияния соседних переключателей. Таким образом, не учитывался возможный межмолекулярный транспорт зарядов в латеральном направлении самого монослоя. Вклад от такого взаимодействия может быть значительным, и очень сложно a priori предположить, будет он увеличивать или уменьшать ON/OFF соотношение для проводимости слоя. На этих задачах будут фокусироваться наши последующие исследования.

Работа выполнена при поддержке Deutsche Forschungsgemeinschaft (DFG), проект GU1510/5-1. Квантово-химические расчеты выполнены на суперкомпьютерах HPC/ZIH “Taurus”, Технический университет Дрездена, и “Glaster”, Институт полимерных исследований научной ассоциации Лейбница, Дрезден.

\section{Список литературы}

1. Orgiu E., Samorì P. // Adv. Mater. 2014. V. 26(12). P. 1827-1845.

2. Russew M. M., Hecht S. // Adv. Mater. 2010. V. 22(31). P. 3348-3360.

3. Ishiguro Y., Hayakawa R., Chikyow T., Wakayama, Y. J. // Mater. Chem. C 2013. V. 1(17). P. 3012-3016.

4. Yagai S., Iwai K., Yamauchi M., Karatsu T. et al. // Angew. Chem. Int. Ed. 2014. V. 53(10). P. 2602-2644.

5. Zhang B., Chen Y., Neoh K.-G., Kang E.-T. Organic Electronic Memory Devices. In Electrical Memory Materials and Devices. 2015. P. 1-53.

6. Van Dyck C., Berger A.J. // Adv. Electron. Mater. 2018. V. 4(5). P. 1700656.

7. Li Y. R., Zhang H. T., Qi C. M., Guo X. F. // J. Mater Chem. 2012. V. 22. P. 4261-4265.

8. Liu Y., Qui X., Soni S., Chiechi R.C. // Chem. Phys. Rev. 2021. V. 2. P. 021303.

9. Karpe S., Oçafrain M., Smaali K., Lenfant S., Vuillaume D., Blanchard P., Roncali J. // Chem. Commun. 2010. V. 46. P. 3657-3659.

10. Smaali K., Lenfant S., Karpe S., Oçafrain M., Blanchard P., Deresmes D., Godey S., Rocherfort A., Roncali J., Vuillaume D. // ACS Nano. 2010. V. 4. P. 2411-2421.

11. Thomas L, Arbouch I., Guérin D., Wallart X., van Dyck C., Melin T., Cornil J., Vuillaume D., Lenfant S. // Nanoscale. 2021. V. 13. P. 6977-6990. 
12. Lenfant S. Charge transport in dynamic molecular junctions. In Molecular Electronics: An Experimental and Theoretical Approach. Pan Stenford Publishing Pte. Ltd.: Singapure, 2016; P. 65-99.

13. Viero Y., Copie G., Guérin D., Krzeminski C., Vuillaume D. Lenfant S., Cleri F. // J. Phys. Chem. C. 2015. V. 119. P. 21173-21183.

14. Viero Y., Guérin D., Vladyka A., Alibart F., Lenfant S., Calame M., Vuillaume D. // Adv. Funct. Mater. 2018. V. 28. P. 1801506.

15. Stiévenard D., Guérin D., Lenfant S., Lévêque G., Nijhuis C. A., Vuillaume D. // Nanoscale. 2018. V. 10. P. 23122-23130.

16. Koch M., Saphiannikova M., Santer S., Guskova O. // J. Phys. Chem. B. 2017. V. 121. P. 8854-8867.

17. Montagna M., Guskova O. // Langmuir. 2018. V. 34. P. 311-321.

18. Loebner S., Lomadze N., Kopyshev A., Koch M., Guskova O., Saphiannikova M., Santer S. // J. Phys. Chem. B. 2018. V. 122. P. 20012009.

19. Koch M., Saphiannikova M., Guskova O. // Langmuir. 2019. V. 35. P. 14659-14669.

20. Savchenko V., Koch M., Pavlov A. S., Saphiannikova M., Guskova O. // Molecules. 2019. V. 24. P. 4387.

21. Grenzer M., Guskova O., Toshchevikov V., Ivaneyko D. // International Innovation. 2016. V. 200. P. 58-60.

22. Gaussian 09, Revision C.01, Gaussian, Inc., Wallingford CT, 2016.

23. Duarte L., Fausto R., Reva I. // J. Comput. Chem. 2014. V. 16. P. 1691916930.

24. Huddleston P. R., Volkov V. V., Perry C. C. // Phys. Chem. Chem. Phys. 2019. V. 21. P. 1344-1353.

25. Guskova O.A. In: Quantum Systems in Physics, Chemistry, and Biology, 2017. P. 209-230.

26. Гуськова О.А., John А. // Вестник ТвГу. Сер. Химия. 2016. Т. 3. С. $42-$ 51.

27. Ye L., Xu C., Gu F.L., Zhu C. // J. Comput. Chem. 2020. V. 41. P. 635-645.

28. Jacquemin D., Wathelet V., Perpete E. A., Adamo C. // J. Chem. Theory Comput. 2009. V. 5. P. 2420-2435.

29. Tomasi J., Mennucci B., Cances E. // J. Mol. Struct. THEOCHEM. 1999. V. 464. P. 211-226.

30. Schuster S., Füser M., Asyuda A., Cyganik P., Terfort A., Zharnikov M. // Phys. Chem. Chem. Phys. 2019. V. 21(18). P. 9098-9105.

31. Diez V., Van Dyck C., Osella S., Cornil D., Corlin J. // Appl. Mater. Interfaces 2021. V. 13. P. 27737-27748.

32. Sato H., Ushiyama S., Sogo M., Aoki M., Shudo K.-i., Sugawara T., Yanagisawa S., Morikawa Y., Masuda S. // Phys. Chem. Chem. Phys. 2012. V. 14. P. 15412-15420.

33. Sachtler W.M.H., Dorgelo G.J.H., Holschr A.A. // Surface Sci. 1966. V. 5(2). P. 221-229.

34. Scheil K., Gopakumar T. G., Bahrenburg J., Temps F., Maurer R. J., Reuter K., Berndt R. // J. Phys. Chem. Lett. 2016. V. 7(11). P. 2080-2084. 
Об авторах:

ГУСЬКОВА Ольга Александровна - кандидат химических наук, научный сотрудник Института полимерных исследований ассоциации Лейбница (Hohe Straße 6, D-01069 Dresden, Deutschland), член Дрезденского центра вычислительного материаловедения Технического университета Дрездена, еmail: guskova@ipfdd.de

САВЧЕНКО Владислав Анатольевич - аспирант второго года обучения Инженерного факультета Технического университета Дрездена (Fakultät für Maschinenwesen, Technische Universität Dresden, Deutschland, 01069 Dresden, Германия), научный сотрудник Института полимерных исследований ассоциации Лейбница, e-mail: savchenko@ipfdd.de

\title{
MOLECULAR SWITCH BASED ON BITHIOPHENE- AZOBENZENE: HOW TO CONTROL CONDUCTANCE THROUGH THE MONOLAYER USING LIGHT
}

\author{
V.A. Savchenko, ${ }^{1}$ O.A. Guskova ${ }^{1,2}$ \\ ${ }^{1}$ Leibniz-Institute of Polymer Research Dresden, Institute of Theory of Polymers, \\ Hohe Str. 6, 01069 Dresden, Germany \\ ${ }^{2}$ Technische Universität Dresden, Dresden Center for Computational Materials \\ Science (DCMS), 01062 Dresden, Germany
}

Molecular switches based on azobenzene $(a z o)$ are defined as light-responsive molecules which can change between two configurational states under light stimuli. Responsive azo monolayers can be used to modulate the work function, i.e. they tune the properties of the interfaces at the electrodes. In this work, we investigate what happens to the structures, electronic properties, and the charge redistribution within azo-bithiophene ( $a z o-b t)$ monolayers depending on the light stimulus using density functional theory. Two types of switches differing in the order of $a z o$ and $b t$ counting from the anchor group are modelled: $a z o-b t$ and $b t$ $a z o$. One of them (bt-azo) is known from the literature, the remaining one is a product of rational design. We describe trans- and cis-isomers for each switch being in a contact with a gold cluster. Our simulations explain a giant ON/OFF conductance ratio upon UV light stimulus by improved electronic coupling between the cis-isomers (ON-state) and the gold cluster. The trans-isomers (OFFstate) of the simulated switches play the role of the insulators. Moreover, we show which molecular properties are enchanced by molecular design. This study opens up new avenues to the development of the innovative design of electrode surface modifications.

Keywords: azobenzene, isomerisation, photosensitivity, conductivity, density functional theory, grafted monolayer, gold cluster $A u_{10}$ 